Rivista di Storia delle Religioni

$10 \mid 2016$

Varia

\title{
Tagewählerei im Alten Ägypten
}

Hemerology in Ancient Egypt

\section{Alexandra von Lieven}

\section{OpenEdition}

\section{Journals}

\section{Edizione digitale}

URL: http://journals.openedition.org/mythos/424

DOI: $10.4000 /$ mythos. 424

ISSN: 2037-7746

\section{Editore}

Salvatore Sciascia Editore

\section{Edizione cartacea}

Data di pubblicazione: 1 décembre 2016

Paginazione: 31-41

ISBN: 978-88-8241-476-4

ISSN: $1972-2516$

\section{Notizia bibliografica digitale}

Alexandra von Lieven, « Tagewählerei im Alten Ägypten », Mythos [En ligne], 10 | 2016, mis en ligne le 24 septembre 2019, consulté le 28 septembre 2019. URL : http://journals.openedition.org/mythos/424 ; DOI : 10.4000/mythos.424 


\section{Tagewählerei im Alten Ägypten*}

\section{Alexandra von Lieven}

\section{Zusammenfassung}

Im Alten Ägypten existierte eine Tradition von Kalendern mit Tagesbewertungen, die es dem Nutzer erlaubte, für jeden Tag und sogar teilweise für unterschiedliche Tageszeiten differenziert zu erfahren, ob es glückverheißend oder eher kontraproduktiv wäre, eine Unternehmung zu beginnen. Ähnliche Listen gab es auch für Monate. Die Bewertungen beruhten unter anderem auf mythischen Ereignissen, die an den jeweiligen Daten stattgefunden haben sollen, weshalb diese Kalender auch wertvolle Quellen zur Mythologie darstellen. In späterer Zeit gehen sie dann auch eine Verbindung mit astrologischen Theorien ein. Das Konzept dieser dies aegyptiaci lebte bis in die Spätantike fort.

\section{Abstract}

In Ancient Egypt, there existed a tradition of calendars containing information on the days' qualities. They allowed a user to find out whether a day or even a particular time of a given day was propitious for certain activities or not. Similar lists existed also for whole months. The ratings often depended from mythological events purportedly having occurred on that date. Thus, such calendars also offer precious information on mythological concepts. Later on, these calendars also make use of astrological theories. In the term dies aegyptiaci, the concept lived on until Late Antiquity.

\section{Schlüsselworte}

Hemerologie - Menologie - Kalender - Astrologie - dies aegyptiaci

\section{Keywords}

Hemerology • menology • calendar • astrology • dies aegyptiaci

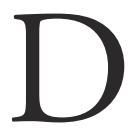
ie Tagewählerei ist in Ägypten eine von zahllosen verschiedenen Divinationstechniken. Im Gegensatz zu der Mehrzahl der anderen hat sie jedoch schon früh einige Beachtung in der Forschung gefunden ${ }^{1}$. Die Quellen liegen, soweit bekannt, fast alle in guten Editionen vor, was leider für andere Zweige der Zukunftsdeutung und „-bewältigung“, wie etwa Astrologie oder Omenkunde, noch immer nicht gelten kann. Das hat wohl nicht zuletzt auch darin seinen Grund, daß die Hemerologie schon historisch recht früh positiv nachweisbar ist.

* Dieser Beitrag wurde ursprünglich am 27. III. 2001 in dem von Joachim Gentz organisierten Panel „Tagewählerei“ auf dem XXVIII. Orientalistentag in Bamberg gehalten. Für die Einladung dazu möchte ich auch an dieser Stelle noch einmal herzlich danken. Weitere Teilnehmer des Panels waren Nils Heeßel und Kocku von Stuckrad. Da sich die ursprünglich geplante Publikation leider nicht materialisiert hat, die Zusammenstellung des Materials aber gerade für Nachbarwissenschaftler immer noch von Interesse sein dürfte, habe ich die Gelegenheit wahrgenommen, den Beitrag in diesem sich thematisch anbietenden Rahmen nun doch noch zu veröffentlichen. Selbstverständlich habe ich die in den vergangenen Jahren erschienene Literatur, soweit erforderlich, eingearbeitet.

1 Bakir 1966, Bács 1987, Troy 1989, Spalinger 1994, Leitz 1994 mit Rezensionen von Quack 1997 und Goyon 1998, Porceddu, Jetsu, MarkKanen, Toivari-Vittala 2008. Der Beitrag Naether, Ross 2008 listet auch einiges kalendarische Material auf, behandelt selbst aber Texte nicht-divinatorischer Natur. 
Mangels ausreichender Bibliotheksfunde muß offenbleiben, wann genau die Tagewählerei erstmals aufkam. Aufgrund des an die Todesprognosen einiger Tage erinnernden Personennamens „Das Krokodil wird sein/ihr Tod sein“ hat Philippe Collombert bereits für das Alte Reich (3. Jtsd.) plädiert² ${ }^{2}$ Der älteste erhaltene Beleg stammt aus dem Mittleren Reich und fand sich unter den sog. Kahunpapyri aus Illahun im Fayum (pKahun XVII.3 = pUC 32192) ${ }^{3}$. Er enthält eine Liste von 30 untereinandergeschriebenen Angaben „guter Tag“ bzw. „schlechter Tag“4, ohne daß im erhaltenen Bereich angegeben wäre, zu welchem Monat die aufgeführten Tage gehören. Der Papyrus war Teil eines großen Fundes dokumentarischen und literarischen Materials und kann etwa in die 12. Dyn. (1976-1794 v. Chr.) datiert werden. Es handelt sich wohl um ein zu persönlichen Zwecken erstelltes Exzerpt aus einem Werk, das sicher auch Begründungen für die Tagesbewertung enthalten haben wird.

Ist der Papyrus aus Illahun nicht sonderlich informativ, so ist es der große Tagewählkalender ḩ.t nhhh ph. wi č.t „Der Anfang ist die Ewigkeit, das Ende ist die Unendlichkeit“ umso mehr ${ }^{5}$. Von diesem Werk sind zwei Abschriften in pKairo JE 86637 und pSallier IV erhalten, davon eine inhaltlich vollständige ${ }^{6}$. Ferner gibt es dazu ein Abrégé in pBM 10474, das nur Datum und Tagesbewertung enthält, ganz so wie pKahun XVII. Dieses datiert etwa in die 26. oder 27. Dyn. (664-401 v. Chr.) ${ }^{7}$. Alle anderen Textzeugen stammen aus dem Neuen Reich (1550-1070 v. Chr.), aus sprachhistorischen Gründen dürfte die Entstehung des Archetyps des großen Tagewählkalenders jedoch ins Mittlere Reich zu datieren $\operatorname{sein}^{8}$. Daneben finden sich im Neuen Reich weitere kleinere Texte, die offensichtlich Auszüge aus größeren Werken darstellen, was zeigt, daß es mehrere verschiedene Kalender gab. Die meisten finden sich ebenfalls in pKairo JE 86637, der Haupthandschrift des Kalenders ḩ.t nhh ph p.wi č.t. Jeweils nur einen Monat enthalten die Ostraka oMalinine und oGardiner 109.

Leider bislang unpubliziert ist der ramessidische pTurin 54022 aus Deir el-Medineh ${ }^{9}$. Dieser enthält dem Vorbericht zufolge nicht nur Tagesbewertungen, sondern gibt auch die zu jedem Tag gehörige Göttin, ihre Tabus sowie Feste $\mathrm{an}^{10}$, was ihm einen noch stärker priesterwissenschaftlichen Charakter gibt, als dem häufiger belegten, für Laien vielleicht relevanteren h̉.t nhh h. ph.wi č.t.

Nicht unerwähnt bleiben soll die bislang einzige belegte Menologie auf einer Schreibtafel der 18. Dyn. (datierbar ca. 1475 v. Chr.), der Text selbst ist wohl ebenfalls um einiges älter und könnte aus sprachhistorischen Gründen tatsächlich schon aus dem Alten Reich (3. Jtsd.) stammen $^{11}$. Der ägyptische Titel lautet 3bty.t, abgeleitet von dem Wort 3bt ,, Monat“.

2 Collombert 2006.

3 Griffith 1898, 62, Pl. XXV, neue Edition bei Collier, Quirke, 2004, 26-27.

4 Unterstreichung hier und im Folgenden markiert im Original rubrizierte Passagen.

5 Ausführlich ediert und kommentiert bei LeITz 1994. Dort S. 1-2 finden sich auch genaue Angaben zu allen in diesem Abschnitt genannten Textzeugen des Neuen Reiches und der Spätzeit. Leider noch immer unpubliziert sind ppTurin 54016, 54022, 54023 und 54029, s. Roccati 1984, S. 348, Anm. 16 und besonders Demichelis 1998, $103-112$. Die ppTurin 54016, 54023 und 54029 sind offenbar weitere Parallelen zu pSallier IV; zu pTurin 54022 s. unten.

6 Leider beinträchtigt die Erhaltung diese Vollständigkeit.

7 Für die Frage der Datierung s. Verhoeven 2001, 290-304.

8 QUACK 1997, 277-278.

9 Demichelis 1998, 103-112, spez. 107-111.

10 Für solche Zeitabschnitten zugeordneten Gottheiten, die sogenannten Chronokratoren, s. YoyotTe 1980, LEITZ 2002, Cauville 2010, Quack 2013, von Lieven (in Druck).

11 Vernus 1981. Zur Datierung s. seine Bemerkungen zur Sprache S. 96, 102-103. 
Im Folgenden ist hauptsächlich die Tagewählerei im engeren Sinne zu behandeln. Infolge der Beleglage läßt sich zu möglichen Entwicklungen der Gattung wenig sagen. Für die Laufzeit ist aber die Frage nach dem Ende der Praxis ebenso relevant wie die nach ihrem Aufkommen. Abgesehen von dem genannten Abrégé der Spätzeit und der etwa zeitgleichen Bemerkung bei Herodot II 83 „Ferner ist von den Ägyptern auch zuerst festgestellt worden, welcher Monat und Tag den einzelnen Göttern heilig ist und welche Schicksale, welches Ende und welchen Charakter die an diesem oder jenem Tage Geborenen haben werden. "12 gibt es weitere Quellen für eine Tradition der Tagewählerei auch noch bis in die griechisch-römische Zeit. Dies sind zunächst Berührungspunkte zum einen mit der Chronokratorenliste aus dem ptolemäischen Tempel von Edfu' ${ }^{13}$, zum anderen mit dem Festkalender aus dem römerzeitlichen Tempel von Esna ${ }^{14}$. Bei beiden Fällen handelt es sich allerdings nicht selbst in toto um Tagewählkalender. Vereinzelte Tagesbewertungen zeigen aber die engen Bezüge. Wahrscheinlich handelt es sich um ein Exzerpt aus einem Werk wie den Berlin-Straßburg-Brooklyner Kultkalendern ${ }^{15}$, die einen Kalender mit Angabe der Schutzgottheiten des jeweiligen Tages, des Tabus der Göttin, des spezifischen Windes, einer zu rezitierenden Schutzbitte für Pharao, eine Tagesbewertung und Angaben über Feste liefern. Die Handschrift Berlin-Brooklyn dürfte aus der Tempelbibliothek von Elephantine stammen und datiert mutmaßlich in die 26. Dyn., die Parallele Berlin-Straßburg kommt eventuell aus dem hermopolitanischen Gau und datiert vermutlich ins 2.-1. Jh. v. Chr. Der Inhalt der beiden Texte ist ganz offensichtlich analog zu pTurin 54022. Wenn dieser einmal vollständig ediert vorliegt, wird es interessant sein, ob es sich um direkte Parallelen und mithin Teile der Tradition eines Textes handelt oder ob zwei verschiedene, nur typologisch analoge Werke vorliegen.

Auch aus dem römerzeitlichen Fayum, namentlich aus Tebtynis und Dime, gibt es relativ umfangreich erhaltene Tempelbibliotheken, die zugleich die wichtigsten Quellen für divinatorische Texte überhaupt sind. In Onomastikon I aus Tebtynis aus dem 2. Jh. n. Chr. konnten ebenfalls leider nur rudimentär erhaltene Reste eines Kalenders mit Tagesbewertungen identifiziert werden ${ }^{16}$. Auch die interessante Notiz über den 3. Tag der Epagomenen bei Plutarch, De Iside $12^{17}$ zeigt, daß seine Informanden noch Zugang zu den traditionellen mythologischen Tagesbewertungen hatten.

Neben diese klassischen Hemerologien alten Stils trat in dieser Zeit jedoch noch ein weiterer, völlig neuartiger Typ. Seit der Spätzeit gewann in Ägypten die Astrologie immer mehr an Gewicht, in hellenistisch-römischer Zeit ist sie dann von zentraler Bedeutung. Betrachtet man etwa den Textbestand in Tebtynis, so wird deutlich, daß v.a. die Astrologie das sprachlich junge (d.h. demotische) divinatorische Material dominiert. Nun richtete sich auch die Bewertung einzelner Zeiträume nach dem Tierkreis und den Planetenständen darin. Weitere Beispiele bereits in griechischer Sprache sind PGM III 275-281 und PGM VIII 284-299, wo bestimmte divinatorische Techniken je nach Zeichen des Zodiakos empfohlen werden, daneben die Kalender der sog. „Laienastrologie“18. Traditionelle Hemerologien waren demgegenüber inzwischen offenbar weniger gefragt, siehe aber immerhin noch PGM VII 158-167.

12 Übersetzung zitiert nach LeITZ 1994, VI.

13 Edfou XV 45-62, Leitz 1994, 2.

14 SPALINGER 1991.

15 QuACK 2012. Die Brooklyner Fragmente der zweiten Berliner Handschrift sind noch unpubliziert.

16 Osing 1998, 207, Taf. 18.

17 Griffiths 1970, 136-137, 305-307. Vgl. dazu die Bemerkungen bei Fischer-Elfert 2006.

18 S. dazu Gundel, Gundel 1966, 272-274, Boll, Bezold, Gundel 19777, 173-183. 
Der Begriff dies aegyptiaci zeigt, daß man noch in der Spätantike Ägypten mit der Tagewählerei verband. Leider ist unklar, ob sich dieser Terminus ursprünglich eher auf die traditionelle oder die astrologisch überformte Tagewählerei richtet. Die Belege sind diesbezüglich wenig aussagekräftig ${ }^{19}$.

Wie sah nun die „klassische“ Tagewählerei der älteren Epochen aus? Als Beispiel sei der große, von Christian Leitz bearbeitete Tagewählkalender herausgegriffen. Wie alle ägyptischen Hemerologien operiert dieser Kalender mit dem 360-Tage-Jahr zu 12 Monaten à je 30 Tagen, verteilt auf die drei am landwirtschaftlichen Zyklus orientierten Jahreszeiten 3h.t, pr.t und šmw. Jeder Monat besteht aus 3 Dekaden, also zehntägigen „Wochen“. Zu diesen 360 Tagen kommen die 5 Zusatztage am Ende eines jeden Jahres hinzu, die sog. Epagomenen. Sie galten als besonders gefährlich und werden daher auch im Tagewählkalender einer Sonderbehandlung unterzogen. Zusätzlich zu den üblichen Einträgen gibt es hier Vermerke über göttliche Namen und Anrufungen mit der Bitte um Schutz. Wer diese kennt, ist vor den Risiken dieser Tage gefeit.

Der Kalender soll von den Göttern selbst verfaßt sein, damit erhält er eine besondere Autorität. Sein Titel lautet:

«Beginn des (Buches) „Der Anfang ist die Ewigkeit, das Ende ist die Unendlichkeit“, verfaßt von den Göttern des Schreines, zusammengestellt von den Göttern der Neunheit, redigiert von der Majestät des Thot im Großen Reichsheiligtum zur Seite des Allherrn, gefunden in der Bibliothek im Hinterhaus der Neunheit.»

Am Beginn ${ }^{20}$ jeden Monats steht „Haus des Re, $\underline{\text { Haus }}$ des Osiris, $\underline{\text { Haus }}$ des Horus.“. Eine mögliche Deutung wäre, daß jede Dekade eines Monats einem Gott zugeordnet werden sollte, sicher ist dies aber nicht.

Unmißverständlich klar ist jedoch, daß man es hier mit einem Kompendium göttlichen Wissens zu tun hat. Tatsächlich reklamiert dieser Text in einer Intensität höchste Autorität für sich, wie kein anderer erhaltener ägyptischer Text. In der Regel reicht ein göttlicher Autor oder die Auffindung durch einen Weisen in einem Tempel. Der Kairener Kalender wartet dagegen gleich mit der Autorschaft der gesamten Spitze des Pantheons und einer Fundnotiz in einem der wichtigsten Tempel des Landes auf. Ob dies auch tatsächlich seiner religiösen oder praktischen Bedeutung entsprach oder nur ein Bedürfnis des Autors oder Kopisten widerspiegelt, sei vorerst dahingestellt.

In diesem Werk finden sich in scriptio continua hintereinander für jeden Tag des Jahres Einträge nach einem festen Schema: Datum, Tagesbewertung, sowie (meistens) eine Angabe über Götterfeste bzw. mythische Ereignisse und/oder Ratschläge für den Benutzer, die in Zusammenhang mit den götterweltlichen Ereignissen und der Tagesbewertung stehen. Fallweise wird angegeben, welches Schicksal der an diesem Tag geborene Mensch haben wird, in diesem Kontext gibt es auch Prognosen zur Todesursache. Die Tagesbewertung ist dreigeteilt, ein Tag besitzt keine einheitliche Qualität, sondern die drei Drittel eines Tages können durchaus unterschiedlich bewertet sein. Als Kategorien stehen „gut“, „ungewiß“ und „gefährlich“ zur Verfügung, wobei die beiden letzteren im Text rubriziert sind. Dies zeigt, daß auch „ungewiß“ tendentiell eher

19 Hopfner 1922-25, 522-528, 561, 573, 647, 528, STERn 1953, 67-68. Für ein koptisches Beispiel s. jetzt Theis in diesem Band.

20 S. dazu Quack 1997, 279. 
etwas Negatives bedeutet, da Rot in Ägypten eine negativ konnotierte Farbe war. Tatsächlich ist auffällig, daß die Fassung des pSallier IV häufig „ungewiß“ einsetzt, wo die Kairener Handschrift „gefährlich“ hat. Berücksichtigt man, daß im Ägyptischen „gefährlich“ “ $h$ ’ mit Aleph heißt, „ungewiß “ aber ${ }^{c} h^{c}$ mit Ayin, so drängt sich der Verdacht auf, daß es sich hier, wie so häufig im ägyptischen Sprachgebrauch, um einen Euphemismus für nicht Genehmes handelt. Vergleichbar ist die Praxis, sprachlich nicht Göttern oder dem Pharao Schlechtes zustoßen zu lassen, sondern deren Feinden, auch wenn der Sinn der Aussage auf das genaue Gegenteil abzielt ${ }^{21}$. In derselben Geisteshaltung verwendet pSallier IV auch einige Male rubriziertes $n f r$,gut", wo die Kairener Parallele ${ }^{C} h$ ' , gefährlich “ schreibt, d.h. pSallier IV hat strenggenommen vier Bewertungen, wobei zwei miteinander identisch sind. Rubriziertes $\underline{n f r}$ tritt jedoch nur insgesamt viermal im Text auf $(1,3 ; 1,5 ; 1,9 ; 11,9)$, davon dreimal auf der ersten Seite. In diesen drei Fällen steht jeweils $\underline{n f r} n f r$

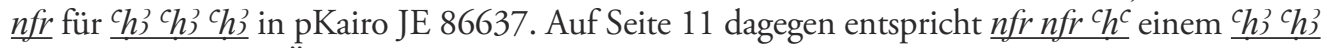
$\underline{(h)}$ in der Parallele. Überall sonst im Text steht ${ }^{C} h$ ) für "gefährlich", es erscheint erstmals in 2,6. Inhaltlich ist kein Grund für einen Unterschied zu erkennen. Will man nicht völlige Willkür annehmen, was kaum plausibel scheint, wäre folgendes Erklärungsmodell denkbar: Der Schreiber hätte in seiner Vorlage einen durchgehend euphemistischen Text (d.h. mit rubriziertem $\underline{n f r}$ für (h) vorliegen gehabt und ihn entsprechend zu kopieren begonnen. Auf der zweiten Seite angelangt, hätte er sich dann entschieden, diese euphemistischen Schreibungen gemäß ihrer wirklichen Bedeutung zu ersetzen. Diesen Entschluß hätte er dann bis auf einen Flüchtigkeitsfehler auf der elften Seite durchgehalten.

Allerdings ist zu den Bewertungen und auch den Begründungen zu bemerken, daß die verschiedenen Textzeugen sich keineswegs stets einig sind. Etwa zum III. 3h.t 18 sind in den drei Textzeugen als Tagesqualität die drei verschiedenen Möglichkeiten zu finden. Dies erschwert natürlich einen Versuch, die Mechanismen der Korrelationen zu entschlüsseln, erheblich. Als Ursachen für solche Diskrepanzen sind ja nicht nur Unaufmerksamkeiten im Tradierungsprozeß anzusetzen, sondern man darf auch die produktive Rezeption und Tradition nicht unterschätzen. Die Gründe für einen Schreiber, in den Textbestand einzugreifen, mögen aber ebenso lokales Sondergut gewesen sein, das vielleicht noch zu fassen sein mag, wie sie sich im Persönlich-Anekdotischen verlieren können. Jeder Versuch einer Interpretation ist daher immer cum grano salis zu beurteilen.

Die Handlungsanweisungen, meist Verbote, beziehen sich nicht nur auf Kultisches, sondern auch auf recht allgemeine Belange des täglichen Lebens, z.B. ob es ratsam sei, irgendeine Unternehmung zu beginnen. Entscheidend ist offenbar nur die Nutzanwendung für den Leser.

Ein typisches Beispiel ist der Eintrag für den IV. 3h.t 21: „4. Monat der Überschwemmungszeit, Tag 21. Gefährlich, gefährlich, gut. Jener Tag des Hervorgehens der Großen aus der Unterwelt, um das Wirksame Auge zu suchen. Du sollst nicht nach draußen gehen am Mittag des Tages."

Die Menologie ist etwas anders organisiert. Jedem Monat einschließlich der fünf Zusatztage wird eine Gottheit zugeordnet und Angaben über Omina gemacht, denen man begegnen kann, z.B.:

21 Posener 1970, Quack 1989. Für einen vergleichbaren Fall s. Quack 1993 (mit Polemik dagegen von Franke 1998, die aber nicht überzeugen kann, etwas differenzierter DepUyd 1998). 
"Zweiter Monat der Überschwemmungszeit. Schentait ist die Wirksame dieses Monats. Wenn du aus deinem Haus herauskommst und jemand neben dir sagt ,Nicht ich bin es, der hitzig ist', oder wenn eine Frau sagt ,Nicht ich bin es, die hitzig ist', dann sollst du dazu sagen ,Wie gut ist dieser Monat! Es wird kein Fieber darin geben.'.»

Meist werden sogar zwei verschiedene Omina genannt, ein gutes und ein schlechtes. Besonders hervorzuheben ist, daß die sprachliche Konstruktion der Prognosen in der Form ir X čt.hr $h r=k Y$,Wenn X (der Fall ist), dann sollst du dazu sagen ,Y'. genau derjenigen entspricht, wie sie auch in wissenschaftlichen Texten für medizinische Diagnosen verwendet wird ${ }^{22}$.

Die interessanteste Frage ist bei Hemerologien und vergleichbaren divinatorischen Texten wie Omenserien u.ä. die nach der Systematik. Im Falle der Menologie ist diese meist leicht durchschaubar, wie in dem zitierten Beispiel ${ }^{23}$. Der große Tagewählkalender ist da weit komplizierter. Hier hat bereits der Herausgeber Leitz umfängliche Untersuchungen vorgelegt ${ }^{24}$.

Er sieht folgende Prinzipien am Werk:

I. Ausdeutung jahreszeitlich abhängiger Naturereignisse,

II. Anknüpfung an bereits bestehende Festdaten,

III. Verbindung zum unmittelbar benachbarten Kalendertag,

IV. Zwei oder mehr Kalendertage bilden eine Einheit,

V. Gleichsetzung von Monatstagen mit Stunden eines Tages,

VI. Gleichsetzung von Monatstagen mit Mondmonatstagen,

VII. Gleiche Kalendertage unterschiedlicher Jahreszeiten sind gleich oder entgegengesetzt konnotiert,

VIII. Kalendertage, die genau ein halbes Jahr voneinander entfernt sind, haben entgegengesetzte Deutung,

IX. Anspielung auf die Zahlen im Datum, und

X. Mehrere Tage sind so miteinander verbunden, daß die Summe ihrer Jahrestage eine markante Zahl ergibt.

Diese Prinzipien wiederum stützen sich auf Leitz' Interpretation der einzelnen Tageseinträge. Diese sind gerade auch naturwissenschaftlich sehr fundiert, doch bestehen im Detail noch einige erhebliche Probleme mit seinen Deutungen. Diese in extenso zu diskutieren wäre lohnend, doch ist hier nicht der geeignete Ort. Um nur ein Beispiel zu nennen, sei darauf hingewiesen, daß Leitz an die Identifikation angeblicher Löwen umfängliche meteorologische Theorien anknüpft, die hinfällig werden, wenn man stattdessen annimmt, daß es sich bei den „Löwen“ ( $m$ 3ỉ) tatsächlich um Schreibungen für $m$ 3) „sehen“ handelt ${ }^{25}$.

Einen sicheren Bezug zwischen Meteorologie und Hemerologie liefert der leider bislang nur durch eine kurze Besprechung bekanntgemachte brontoskopische Kalender pTurin 54024 aus dem ramessidischen Deir el-Medineh ${ }^{26}$. Er gehörte offenbar zu einem größeren Konvolut

22 Vgl. Quack 2010 zu Ägypten und Althoff 2010 zu Griechenland.

23 Vernus 1981, 104.

24 Neben dem Kommentar in Leitz 1994 ist auch Leitz 1997 zu nennen.

25 Bereits gesehen von QuAck 1997, 281-282, der auch einige weitere Probleme anspricht.

26 Kurze Beschreibung und Diskussion bei Roccati 1994, spez. 347-351. Interessant für die eventuelle Laufzeit solcher Konzepte in Ägypten ist der Text, auf den RoccATI S. 350 zum Vergleich verweist, da sein Gewährsmann Johannes Laurentius Lydus des 7. Jh. n. Ch. seine Angaben auch noch „ex Aegyptiorum doctrina“ bezogen haben will. 
divinatorischer Werke, zu dem mindestens auch ein lekanomantisches Handbuch, ein Buch über Erdbebenomina und ein Buch über Vorhersagen im Zusammenhang der Gründung eines Hauses gehörten ${ }^{27}$. Dem Vorbericht zufolge finden sich in diesem Werk für jeden Tag des Jahres Einträge im Stile von "Was angeht, wenn Seth die Stimme hören läßt im Himmel ${ }^{28}$ im Monat x der Jahreszeit y, Tag $\mathrm{z}$, dann sollst du dazu sagen: ...“. Auch hier wird also das klassische Diagnoseformular wie in den medizinischen Texten verwendet.

Die Tagewählerei ist, wie erwähnt, in Ägypten nur eine der vielen Divinationstechniken, derer man sich bedienen konnte, um sich im Leben zurechtzufinden. Tatsächlich besaß Ägypten eine reiche Divinationskultur, deren Schätze noch kaum gehoben sind ${ }^{29}$. So lassen sich fast alle divinatorischen Techniken nachweisen: Orakel, Omina jeglicher Couleur, verschiedene Arten von Mantik, Traumdeutung und Astrologie.

Die Tagewählerei steht also nicht vereinzelt. All diesen Techniken liegt die Auffassung zugrunde, man könne durch entsprechendes Wissen um die zukünftigen Dinge möglicherweise drohendem Unheil aus dem Wege gehen. Unmittelbar leuchtet dies ein bei Handlungsanweisungen, wo man durch Tun oder Unterlassen bestimmter Tätigkeiten den Ausgang der Situation selbst in der Hand hat. Wer zu Hause bleibt, wenn Dämonen auf der Erde herumschleichen, läuft natürlich weniger Gefahr, von ihnen getötet zu werden. Was aber ist zu tun, wenn, wie am I. 3h.t 16, dem an jenem Tag Geborenen der Tod durch ein Krokodil prophezeit wird? Diese Information wirkt zunächst unumstößlich und folglich eher deprimierend. Eine ähnliche Prophezeiung erhält ein Prinz in einer neuägyptischen Erzählung in die Wiege gelegt, allerdings durch direkte göttliche Mitteilung: die sieben Hathoren verkünden bei der Geburt des Prinzen seinen künftigen Tod durch ein Krokodil, eine Schlange oder einen Hund ${ }^{30}$. Leider ist der Text nicht vollständig erhalten, so daß offen bleiben muß, auf welche Weise der Held seinem Schicksal entgeht. Daß er überlebt, ist jedoch sehr wahrscheinlich, da ägyptische Erzählungen ein Happy End bevorzugen und der ganze Text einen recht märchenhaften Charakter aufweist. Während der Prinz in der Erzählung offenbar vor allem in seiner Frau eine tatkräftige Unterstützung gegen die Fährnisse des Lebens findet, bevorzugte der real existierende Ägypter göttlichen Beistand. Eine ganze Anzahl von Orakeldekreten ${ }^{31}$ hat sich erhalten, in denen Götter versprechen, den Besitzer vor allem Übel zu schützen. Einige dieser Amulette offenbaren auch eine wenig soziale Art der Zukunftsbewältigung: ähnlich dem altorientalischen Ersatzkönigsbrauch verspricht der jeweilige Gott seinem Schützling, einen anderen Menschen an seiner Stelle das verhängte Schicksal erleiden zu lassen. Umgekehrt versichert er folgerichtig, den Betreffenden aber niemals für einen Anderen als Substitut einzusetzen. Offenbar ging man also davon aus, daß das göttlich festgesetzte Schicksal zwar zwangsläufig eintreffen müsse, aber noch Spielraum bestünde, wen es letztendlich träfe. Es ist daher kein Zufall, daß (besonders in der Spätzeit) die Verehrung der Schicksalsgötter Schai ${ }^{32}$

27 Demichelis 2002, 151, 159 mit Anm. 6. Zu demselben Konvolut gehört wohl auch der bei RocCATi 1994 behandelte Text mit offenbar stundenweise differenzierten Anfragen an den Sonnengott und die schon erwähnte Hemerologie pTurin 54016.

28 D.h. wenn es donnert. Seth galt u.a. als Wettergott.

29 S. in extenso von Lieven 1999, seitdem ist eine Fülle weiterer wichtiger Quellen bekannt geworden, davon sei neben Demichelis 2002 als Überblick nur QuACK 2006 und ders., in diesem Band genannt.

30 Übersetzung mit Literaturverweisen bei LiсHтHEIM 1976, 200-203.

31 EDWARDS 1960.

32 Quaegebeur 1975. 
und Renenet blühte und Ägypten in griechisch-römischer Zeit ein Zentrum des Kultes der mit Schai assoziierten Nemesis ${ }^{33}$ wurde.

Obwohl solche Orakeldekrete erst seit dem Ende des Neuen Reiches bezeugt sind, darf man annehmen, daß es auch vorher bereits Methoden gab, schlechten Zukunftsprognosen aktiv entgegenzuwirken ${ }^{34}$.

Der Ägypter hatte also allen Grund, genau in einen Tagewählkalender zu schauen. Die Frage ist nur: Tat er es auch? Hier ist man zwangsläufig auf schlüpfrigem Datengrund. Deshalb sollte man Rosemarie Drenkhahns Feststellung, die Tagewählkalender hätten kaum praktische Relevanz im täglichen Leben gehabt ${ }^{35}$, auch nicht überbewerten. Bedenkenswert ist ihre Untersuchung aber allemal. Sie verglich nämlich die Tageswertungen in den Kalendern mit den Daten erhaltener Geschäftsakten und königlicher Verlautbarungen und stellte fest, daß sich an Tagen, die als gefährlich vermerkt oder gar für alle Arbeit untauglich erklärt waren, durchaus keine Abstinenz von Alltagsgeschäften finden ließ. Andererseits konnte Leitz für zwei kleinere Exzerpte, pKairo JE 86637 vso. XII und oGardiner 109 darauf hinweisen, daß für bestimmte Tage vom Gang zur knb.t, der Ratsversammlung, abgeraten wird, und tatsächlich für die betreffenden Daten keine Sitzung dieser Versammlung aus den erhaltenen Verwaltungsdokumenten belegt is $\mathrm{t}^{36}$.

Zu dieser Diskrepanz der Ergebnisse ist zu bemerken, daß das erhaltene Aktenmaterial natürlich nicht repräsentativ ist, wie erwähnt, nicht einmal die einzelnen Kalendertextzeugen sich immer einig sind, man nicht davon ausgehen kann, daß die Person, deren Rechnungen man vor sich hat, auch wirklich diesen, oder überhaupt einen Tagewählkalender benutzt hat, etc.

Besitz und Lektüre eines solchen Kalenders sind auch nur in bestimmten Bevölkerungssegmenten plausibel. Leider wissen wir von den wenigsten hemerologischen Texten wirklich sicher, wo sie gefunden wurden, und damit, aus welchem sozialen Milieu sie stammen. In den Fällen, wo die Provenienz bekannt oder zumindest halbwegs plausibel vermutet ist, handelt es sich im Neuen Reich um Deir el-Medineh. Da die dort lebenden Arbeiter aufgrund ihrer Tätigkeit am Königsgrab aber nicht so ohne weiteres repräsentativ sind, ist eine Verallgemeinerung problematisch. Der einzig positive Beleg aus dem Mittleren Reich stammt aus der Siedlung von Illahun. Genauere Angaben zu den konkreten Häusern, in denen die Texte gefunden wurden, fehlen leider in beiden Fällen, was die wirklich soziologische Aussagekraft dieser Provenienzangaben leider massiv limitiert.

Der Verdacht, daß auch im Fall der Tagewählerei, wie in verschiedenen anderen Bereichen der ägyptischen Religion ein Brauch aus dem Tempel auf die weitere Oberschicht ausgreift und dann langsam auch den Rest der Bevölkerung erfaßt, liegt nahe. Wenn I.E.S. Edwards ${ }^{37}$ annimmt, die Orakeldekrete stammten von einfachen Leuten, da keine Titel genannt werden, so ist dies wenig überzeugend. Tatsächlich spielen im Verhältnis zur Gottheit offenbar die Titel keine Rolle, es reicht, ein „Diener“ dieser Gottheit zu sein. Für die Abwesenheit von Titeln ist auch ins Feld zu führen, daß Orakeldekrete möglicherweise für Kinder erteilt wurden, die natürlich noch keine langen Titelreihen hatten. Immerhin handelt es sich in den Fällen, wo der soziale Status bekannt ist, durchweg um Angehörige der Priesterschaft bzw. in einem Falle sogar um einen Königssohn.

33 LichOCKa 2004.

$34 \mathrm{Zu}$ diesem Komplex vgl. im Detail den Beitrag von QuACK in diesem Band.

35 DrenkHAhN 1972. Immerhin überwiegen die positiven Daten etwas.

36 Leitz 1994, 448-451 mit Anm. n (S. 449).

37 EDWARDs 1960, xv-xvi. 
Tatsächlich war eine große Zahl erhaltener divinatorischer Texte sicherer Provenienz - etwa das späte Material aus Tebtynis - definitiv im Umfeld von Tempelbibliotheken angesiedelt. Die aus der abendländischen Definition von „Aberglauben“ erwachsene Vorstellung, solche Praktiken entstammten einem diffusen und naiven „Volksglauben“, ist für Ägypten schlicht unhaltbar. Das soll nun freilich nicht heißen, daß das Volk sich nicht für Zukunftsdeutung interessiert habe. Doch in den unteren Bevölkerungsschichten wird man in viel stärkerem Maß mit oraler Tradition rechnen müssen, die für die Forschung für immer verloren ist.

Welche Rolle die Tagewählerei also wirklich im Alltagsleben spielte, ist nicht so leicht zu entscheiden. Insgesamt wird man dem Phänomen vermutlich am ehesten gerecht, wenn man akzeptiert, daß sich echter Glaube und lebensnaher Pragmatismus nicht ausschließen (müssen). Die für Ägypten so oft attestierte „Multiplicity of approaches“ darf man wohl auch für die gesellschaftliche Relevanz der Tagewählerei in Rechnung stellen.

Alexandra von Lieven

Ägyptologisches Seminar

Freie Universität Berlin

Fabeckstr. 23-25

14195 Berlin

alexandra.von.lieven@fu-berlin.de

\section{Bibliographie}

\section{Althoff 2010}

J. Althoff, "Das Verhältnis von medizinischer Prognose zur religiösen Divinatorik/Mantik in Griechenland", in A. Imhausen, T. Pommerening (Hg.), Writings of Early Scholars in the Ancient Near East, Egypt, Rome, and Greece (Beiträge zur Altertumskunde 286), Berlin 2010, 47-68.

BÁCs 1987

T.A. Bács, «Prolegomena to the Study of Calenders of Lucky and Unlucky Days», in A. Roccati (Hg.), La magia in Egitto ai tempi dei faraoni, Mailand 1987, 245-256.

BAKIR 1966

A. Bakir, The Cairo Calendar, Kairo 1966.

Boll, Bezold, Gundel $1977^{7}$

F. Boll, C. Bezold, W. Gundel, Sternglaube und Sterndeutung. Die Geschichte und das Wesen der Astrologie, Stuttgart $1977^{7}$.

Cauville 2010

S. Cauville, «La permanence de la protection (à propos des "chronocrates")", Journal of the American Research Center in Egypt 46 (2010), 233-273.

Cauville, Devauchelle 1985

S. Cauville, D. Devauchelle, Le temple d'Edfou XV, Kairo 1985.
Collier, Quirke 2004

M. Collier, S. Quirke, The UCL Lahun Papyri: Religious, Literary, Legal, Mathematical and Medical (British Archaeological Reports S1209), Oxford 2004.

Collombert 2006

P. Collombert, "Un étrange anthroponyme de l'Ancien Empire: "Il/Elle mourra par le crocodile " (?)», Göttinger Miszellen 209 (2006), 33-42.

Demichelis 1998

S. Demichelis, «Papiri calendariali del Museo Egizio di Torino", Vicino Oriente 11 (1998), 103-112.

Demichelis 2002

S. Demichelis, "La divination par l'huile à l'époque ramesside», in Y. Koenig (Hg.), La magie en Égypte: à la recherche d'une définition, Paris 2002, 149-165.

Depuydt 1998

L. Depuydt, "Far Towards": A Common Hieroglyphic Idiom», Journal of Ancient Civilizations 13 (1998), 39-46.

DRENKHAHN 1972

R. Drenkhahn, "Zur Anwendung der „Tagewählkalender"", Mitteilungen des Deutschen Archäologischen Instituts in Kairo 28 (1972), 85-94.

EDWARDS 1960

I.E.S. Edwards, Oracular Amuletic Decrees of the Late 
New Kingdom (Hieratic Papyri in the British Museum $4^{\text {th }}$ Series), London 1960.

Fischer-ELFERT 2006

H.-W. Fischer-Elfert, "Ammunenshi und die Tagewählerei oder Der präsumtive Todestag Amenemhets' I. (Sinuhe B 43-45 und R 5-6)", in H.-W. FischerElfert, K. Zibelius-Chen (Hg.), „Von reichlich ägyptischem Verstande". Festschrift für Waltraud Guglielmi (Philippika 11), Wiesbaden 2006, 23-27.

Franke 1998

D. Franke, «Das Entfernen eines Sprachtabus. Nochmals zur Konstruktion w3j r», Göttinger Miszellen 165 (1998), 51-56.

GOYON 1998

J.-C. Goyon, «Rezension zu C. Leitz, Tagewählerei.

Das Buch hỉt nḥh ph.wy $d t$ und verwandte Texte (Ägyptologische Abhandlungen 55), Wiesbaden 1994», Bibliotheca Orientalis 55 (1998), Sp. 747-753.

GRIFFITH 1898

F.Ll. Griffith, Hieratic Papyri from Kahun and Gurob (principally of the Middle Kingdom), London 1898.

Griffiths 1970

J.G. Griffiths, Plutarch's De Iside et Osiride, Cardiff 1970 .

Gundel, Gundel 1966

W. Gundel, H. Gundel, Astrologumena (Sudhoffs Archiv, Beiheft 6), Wiesbaden 1966.

HopfNer 1922-25

Th. Hopfner, Fontes historiae religionis aegyptiacae, Bonn 1922-25.

\section{LEITZ 1994}

C. Leitz, Tagewählerei. Das Buch hỉt nhh ph.wy $\underline{d} t$ und verwandte Texte (Ägyptologische Abhandlungen 55), Wiesbaden 1994.

\section{LEITZ 1997}

C. Leitz, «Die Erweiterung der Mythen um eine kalendarische Dimension - zur Systematik altägyptischer Hemerologien", Kodikas/Code. Ars Semeiotica Volume 20, No. 1-2 (1997), Special Issue: Kult, Kalender und Geschichte, 39-51.

LEITZ 2002

C. Leitz, «Die Chronokratenliste aus Edfu - ein Pantheon aus der zweiten Hälfte der 12. Dynastie», Revue d'Égyptologie 53 (2002), 143-162.

LiCHOCKA 2004

B. Lichocka, Némésis en Égypte Romaine (Aegyptiaca Treverensia 5), Mainz 2004.
Lichtheim 1976

M. Lichtheim, Ancient Egyptian Literature II, Berkeley/Los Angeles/London 1976.

VON LIEVEN I 999

A. von Lieven, «Divination in Ägypten», Altorientalische Forschungen 26 (1999), 77-126.

von Lieven (IN Druck)

A. von Lieven, "Divine Figurations of Time in Ancient Egypt», in L. Doering, J. Ben-Dov (Hg.), The Construction of Time in Antiquity, Cambridge (in Druck).

NAETHer, Ross 2008

F. Naether, M. Ross, «Interlude: a Series Containing a Hemerology with Lengths of Daylight», Egitto e Vicino Oriente 31 (2008), 59-90.

OsING 1998

J. Osing, Hieratische Papyri aus Tebtunis I (The Carlsberg Papyri 2, Carsten Niebuhr Institute Publications 17), Kopenhagen 1998.

Porceddu, Jetsu, Markkanen, Toivari-Vittala 2008 S. Porceddu, L. Jetsu, T. Markkanen, J. Toivari-Viitala, «Evidence of Periodicity in Ancient Egyptian Calendars of Lucky and Unlucky Days", Cambridge Archaeological Journal 18 (2008), 327-339.

Posener 1970

G. Posener, "Sur l'emploi euphémique de $h f t j(w)$ "ennemi(s)"", Zeitschrift für ägyptische Sprache und Altertumskunde 96 (1970), 30-35.

QUACK 1989

J.F. Quack, «Sur l'emploi euphémique de $h f t$ «ennemi» en Démotique», Revue d'Égyptologie 40 (1989), 197-198.

Quack 1993

J.F. Quack, «Ein altägyptisches Sprachtabu», Lingua Aegyptia 3 (1993), 59-79.

QuAck 1997

J.F. Quack, «Rezension zu C. Leitz, Tagewählerei. Das Buch hỉt nhh ph.wy $\underline{d} t$ und verwandte Texte, (Ägyptologische Abhandlungen 55), Wiesbaden 1994", Lingua Aegyptia 5 (1997), 277-287.

QuACK 2006

J.F. Quack, "A Black Cat from the Right, and a Scarab on your Head. New Sources for Ancient Egyptian Divination", in K. Szpakowska (Hg.), Through a Glass Darkly: Magic, Dreams, and Prophecy in Ancient Egypt, Swansea 2006, 175-187.

QuACK 2010

J.F. Quack, «Präzision in der Prognose oder: Divination als Wissenschaft», in A. Imhausen, T. Pommere- 
ning (Hg.), Writings of Early Scholars in the Ancient Near East, Egypt, Rome, and Greece (Beiträge zur Altertumskunde 286), Berlin 2010, 69-91.

\section{QuACK 2012}

J.F. Quack, «Reste eines Kultkalenders (pBerlin $14472+$ pStrasbourg BNU hier. 38 a und pBerlin 29065", in V.M. Lepper (Hg.), Forschungen in der Papyrussammlung. Eine Festgabe für das Neue Museum (Ägyptische und Orientalische Papyri und Handschriften des Ägyptischen Museums und Papyrussammlung Berlin 1), Berlin 2012, 189-206

QuACK 2013

J.F. Quack, «Zeit, Krise und Bewältigung: Ägyptische Zeiteinheiten, ihre Schutzgötter und deren bildliche Umsetzung», in Th. Greub, D. Boschung (Hg.), Das Bild der Jahreszeiten im Wandel der Kulturen und Zeiten (Morphomata 7), München 2013, 73-98.

Quaegebeur 1975

J. Quaegebeur, Le dieu égyptien Shaï dans la religion et l'onomastique (Orientalia Lovaniensi Analecta 2), Leuven 1975.

\section{Roccati 1984}

A. Roccati, "Lessico Meteorologico», in F. Junge (Hg.), Studien zu Sprache und Religion Ägyptens I. Zu Ehren von Wolfhart Westendorf überreicht von seinen Schülern und Freunden, Göttingen 1984, 343-354.

RocCATI 1994

A. Roccati, "五 Clerc, N. Grimal (Hg.), Hommages à Jean Leclant I (Bibliothèque d'Étude 106/1), Kairo 1994, 493-497.

SPALINGER 1991

A. Spalinger, "An Unexpected Source in a Festival Calendar", Revue d'Égyptologie 42 (1991), 209-222.
SPALINGER 1994

A. Spalinger, "Calendars: Real and Ideal», in B.M. Bryan, D. Lorton (Hg.), Essays in Egyptology in Honor of Hans Goedicke, San Antonio 1994, 297-308.

STERN 1953

H. Stern, Le calendrier de 354. Étude sur son texte et ses illustrations (Institut Français d'Archéologie de Beyrouth, Bibliothèque archéologique et historique 55), Paris 1953

TROY 1989

L. Troy, "Have a Nice Day!», in G. Englund (Hg.), The Religion of the Ancient Egyptians. Cognitive structures and popular expressions. Proceedings of symposia in Uppsala and Bergen, 1987 and 1988 (Acta Universitatis Upsaliensis Boreas, Uppsala Studies in Ancient Mediterranean and Near Eastern Civilizations 20), Uppsala 1989, 127-147.

VERHOEVEN 2001

U. Verhoeven, Untersuchungen zur späthieratischen Buchschrift (Orientalia Lovaniensia Analecta 99), Leuven 2001.

VERNUS 1981

P. Vernus, «Omina calendériques et comptabilité d'offrandes sur une tablette hiératique de la XVIII ${ }^{e}$ dynastie», Revue d'Égyptologie 33 (1981), 89-124.

\section{YoyotTe 1980}

J. Yoyotte, "Une monumentale litanie de granit: les Sekhmet d'Aménophis III et la conjuration permanente de la déesse dangereuse», Bulletin de la Société Française d'Égyptologie 87-88 (1980), 46-75. 